\title{
Type IV Ehlers-Danlos Syndrome: A Surgical Emergency? A Case of Massive Retroperitoneal Hemorrhage
}

\author{
Stephen G. Chun ${ }^{1,2, ;, *}$, Patrick Pedro ${ }^{1, \dagger}$, Mihae $\mathrm{Yu}^{1}$ and Danny M. Takanishi ${ }^{1}$ \\ ${ }^{I}$ Department of General Surgery, John A. Burns School of Medicine, University of Hawaii at Manoa, Honolulu, HI \\ ${ }^{2}$ Cancer Research Center of Hawaii, University of Hawaii at Manoa, Honolulu, HI
}

\begin{abstract}
Retroperitoneal hemorrhagic bleeding is a known manifestation of Type-IV Ehlers-Danlos Syndrome that is caused by loss-of-function mutations of the pro-alpha-1 chains of type III pro-collagen (COL3AI) resulting in vascular fragility. A number of previous reports describe futile surgical intervention for retroperitoneal bleeding in Type-IV Ehlers-Danlos Syndrome with high post-operative mortality, although the rarity of retroperitoneal bleeding associated with Type-IV Ehlers-Danlos Syndrome precludes an evidence-based approach to clinical management. We report a 23-year-old male with history of Type-IV Ehlers-Danlos Syndrome who presented with severe abdominal pain and tachycardia following an episode of vomiting. Further work-up of his abdominal pain revealed massive retroperitoneal bleeding by CT-scan of the abdomen. Given numerous cases of catastrophic injury caused by surgical intervention in Type-IV Ehlers-Danlos Syndrome, the patient was treated non-operatively, and the patient made a full recovery. This case suggests that even in cases of large retroperitoneal hemorrhages associated with Ehlers-Danlos Syndrome, it may not truly represent a surgical emergency.
\end{abstract}

Keywords: Type-IV Ehlers-Danlos Syndrome, retroperitoneal hemorrhage, conservative management, non-operative, COL3A1, connective tissue disorder.

\section{INTRODUCTION}

Retroperitoneal hemorrhagic bleeding is a known manifestation of Type-IV Ehlers-Danlos Syndrome that is caused by loss-of-function mutations of the pro-alpha- 1 chains of type III pro-collagen (COL3AI) resulting in vascular fragility and the hemorrhage of medium sized blood vessels. Due to the vascular frailty associated with Type IV Ehlers-Danlos Syndrome, surgical intervention carries a high risk and is associated with significant morbidity and mortality. Previously, a manuscript was published in the Postgraduate Medical Journal by Soonawalla et al. (2002) entitled "Type IV Ehlers-Danlos Syndrome: A surgical Emergency" [1]. describing a 35-year-old male with Type-IV (vascular subtype) Ehlers-Danlos Syndrome, who developed a retroperitoneal hemorrhage secondary to vomiting. The patient was taken emergently to the operating room because of tachycardia and evidence of massive retroperitoneal bleeding as visualized by an abdominal CT-scan. Adequate hemostasis was achieved intra-operatively, but the patient developed a sigmoid perforation post-operatively. A repeat exploratory laparotomy to repair the sigmoid colon was performed, but the patient unfortunately died of a myocardial infarction following the second operation. The case reported by Soonawalla et al. was similar to other cases in the literature describing

*Address correspondence to this author at the Department of Surgery, John A. Burns School of Medicine University of Hawaii at Manoa 1356 Lusitana Street, University Tower $6^{\text {th }}$ Floor Honolulu, HI 96813; Tel: 214-645-8525; Fax: 214-645-8526; E-mail: Stephen.Chun@UTSouthwestern.edu

$\dagger$ These authors contributed equally to the production of this manuscript. catastrophic results of surgical intervention in patients with Type-IV Ehlers-Danlos Syndrome resulting in high postoperative mortality $[2,3]$. In response to the manuscript by Soonawalla et al. [1] we report a very similar case of TypeIV Ehlers-Danlos associated with retroperitoneal bleeding that we successfully managed non-operatively.

\section{CASE REPORT}

A 23-year-old male with history of Type-IV EhlersDanlos Syndrome and hemorrhagic stroke presented with severe abdominal pain following an episode of vomiting that was precipitated by alcohol consumption. Like the case reported by Soonawalla et al. [1] the patient was tachycardic on initial presentation suggesting hypovolemia, and an abdominal CT-scan revealed a large retroperitoneal hemorrhage (Fig. 1). Angiographic evaluation using interventional radiology was considered for evaluation of this patient's hemorrhage, but was decided against due to the high risk of dissection from arterial catheters in Type IV Ehlers-Danlos Syndrome. Furthermore, based upon the catastrophic consequences of exploratory laparotomy in the case reported by Soonawalla et al. [1] we elected for a trial of conservative management. The patient's initial Hgb was 15.9 that fell to 11.8 on his first hospital day, and then stabilized. For the first 48 hours, the patient was placed on strict bed-rest to prevent any further exacerbation of the hemorrhage, and administered anti-emetics liberally to prevent any further episodes of emesis. Throughout the rest of his hospital stay, the patient's pain improved, Hgb remained stable, and he was discharged on his fourth hospital day. Currently, the 
patient continues to do well nearly one year later and has returned to his usual state of health. To our knowledge, this is the first patient with Type IV Ehlers-Danlos Syndrome to survive the development of a massive retroperitoneal hemorrhage.

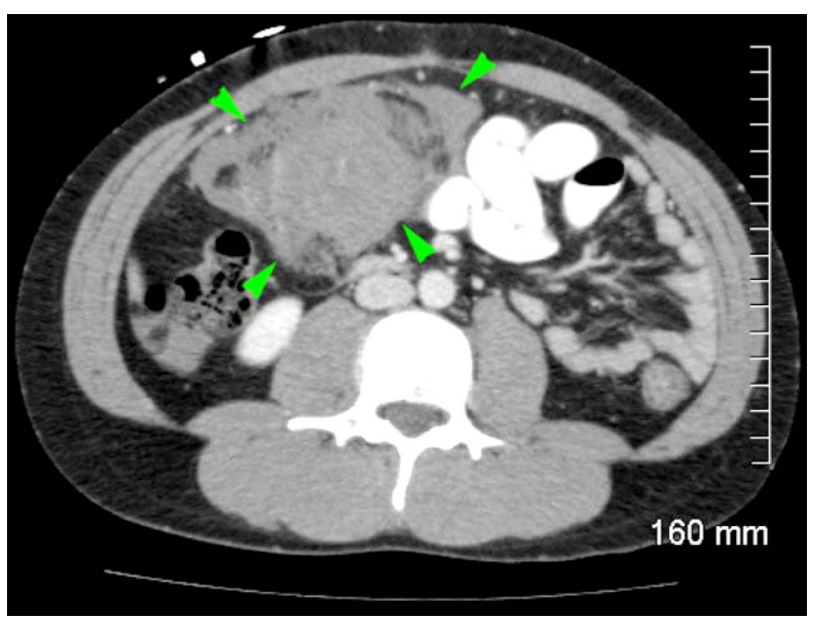

Fig. (1). A patient with a history of Type-IV Ehlers-Danlos Syndrome developed severe abdominal pain secondary to vomiting, and an abdominal CT-scan to work-up the patient's symptoms revealed a large retroperitoneal hemorrhage. Green arrows identify the extent of the hemorrhage from the retroperitoneum into the mesentery on an axial slice of the abdomen by CT-scan.

\section{DISCUSSION}

Type-IV Ehlers-Danlos Syndrome is a rare connective tissue disorder caused by loss-of-function mutations of the gene $C O L 3 A 1$ that encodes the pro- $\alpha-1$ chains of type- 3 procollagen that causes vascular fragility especially of mediumsized blood vessels, and retroperitoneal bleeding is a known complication of this syndrome [4]. While the rarity of this syndrome precludes an evidence-based approach to management, previous cases can serve as a guide in the clinical care of patients with Type-IV Ehlers-Danlos Syndrome [1, 5]. We submit this case as a response to Soonawalla et al. [1] as our case suggests that in such patients, a trial of conservative management instead of emergent surgical intervention may represent a reasonable clinical approach on an ad hoc basis. The operative and post-operative mortality rate for surgical intervention is high in Type-IV Ehlers-Danlos
Syndrome, [6-8] and invasive interventions should be considered as a last resort.

\section{ACKNOWLEDGMENTS}

We thank Dr. Laurie Seaver, M.D., of the Department of Pediatrics of the John A. Burns School of Medicine of the University of Hawaii at Manoa for her helpful discussion in the clinical management of this patient. We also appreciate the assistance of Dr. Shirley Domingo, M.D., and Dr. Dean Sato, M.D. for providing clinical care of this patient. S.G.C. is a Surgical Intern of the Department of General Surgery at the John A. Burns School of Medicine.

\section{DISCLOSURES}

The authors have no financial conflicts of interest to disclose.
ABBREVIATIONS
CT-scan $=\chi$-ray based computed tomographic scan
COL3A1 = Gene encoding the pro- $\alpha-1$ chains of type- 3 collagen
$\mathrm{Hgb} \quad=$ Hemoglobin

\section{REFERENCES}

[1] Soonawalla Z, Pope FM, Puntis M. Type IV Ehlers-Danlos syndrome: a surgical emergency. Postgrad Med J 2002; 78: 501-2.

[2] Horowitz MB, Purdy PD, Valentine RJ, Morrill K. Remote vascular catastrophes after Neurovascular Interventional Therapy for Type 4 Ehlers-Danlos Syndrome. AJNR Am J Neuroradiol 2000; 21 : 974-6.

[3] Berney T, La Scala G, Vettorel D, et al. Surgical pitfalls in a patient with type IV Ehlers-Danlos syndrome and spontaneous colonic rupture. Report of a case. Dis Colon Rectum 1994; 37 : 1038-42.

[4] Pepin M, Schwarze U, Superti-Furga A, Byers PH. Clinical and genetic features of Ehlers-Danlos syndrome type IV, the vascular type. N Engl J Med 2000; 342: 673-80.

[5] Im JS, Lim YH, Park JS, Lee SS, Kim KM. Rupture of abdominal aortic aneurysm after spine surgery in the patient with EhlersDanlos Syndrome. Korean J Anesthesiol 2010; 58: 555-9.

[6] Hosaka A, Miyata T, Shigematsu H, Deguchi JO. Spontaneous mesenteric hemorrhage associated with Ehlers-Danlos syndrome. J Gastrointest Surg 2006; 10: 583-5.

[7] Oderich GS, Panneton JM, Bower TC, et al. The spectrum, management and clinical outcome of Ehlers-Danlos syndrome type IV: a 30-year experience. J Vasc Surg 2005; 42: 98-106.

[8] Jiminez R, Minano-Perez JA, Bercial-Arias J, et al. Ehlers-Danlos tipo IV con complicaciones vasculares precoces. Descripcion de un caso y revision bibliografica. Angiologia 2004; 56: 521-7. 